PROCEEDINGS OF THE

AMERICAN MATHEMATICAL SOCIETY

Volume 135, Number 10, October 2007, Pages 3217-3224

S 0002-9939(07)08847-8

Article electronically published on May 14, 2007

\title{
A STRONG COMPARISON PRINCIPLE FOR THE $p$-LAPLACIAN
}

\author{
PAOLO ROSELLI AND BERARDINO SCIUNZI \\ (Communicated by David S. Tartakoff)
}

\begin{abstract}
We consider weak solutions of the differential inequality of pLaplacian type

$$
-\Delta_{p} u-f(u) \leq-\Delta_{p} v-f(v)
$$

such that $u \leq v$ on a smooth bounded domain in $\mathbb{R}^{N}$ and either $u$ or $v$ is a weak solution of the corresponding Dirichlet problem with zero boundary condition. Assuming that $u<v$ on the boundary of the domain we prove that $u<v$, and assuming that $u \equiv v \equiv 0$ on the boundary of the domain we prove $u<v$ unless $u \equiv v$. The novelty is that the nonlinearity $f$ is allowed to change sign. In particular, the result holds for the model nonlinearity $f(s)=s^{q}-\lambda s^{p-1}$ with $q>p-1$.
\end{abstract}

\section{INTRODUCTION AND STATEMENT OF THE RESULTS}

Throughout this article $\Omega$ will be a bounded smooth domain of $\mathbb{R}^{N}$ with $N \geq 2$. A function $w \in C^{1, \alpha}(\bar{\Omega})$ (see [6, 8, 12]) solves the equation

$$
-\Delta_{p} w=f(w) \text { weakly on } \Omega
$$

(where $p>1$ and $f$ is a continuous real function that is locally Lipschitz on its domain) if and only if

$$
\int_{\Omega}|\nabla w|^{p-2} \nabla w \cdot \nabla \phi d x=\int_{\Omega} f(w) \phi d x \quad \forall \phi \in W_{0}^{1, p}(\Omega) .
$$

In this paper we consider the following problem:

$$
\left\{\begin{aligned}
-\Delta_{p} w & =f(w) & & \text { weakly on } \Omega, \\
w & >0 & & \text { on } \Omega, \\
w & =0 & & \text { on } \partial \Omega .
\end{aligned}\right.
$$

We restrict our attention to the case of positive solutions, and we recall that by the strong maximum principle for the p-Laplacian under quite general hypotheses on $f$ (see [10, 13]) any nonnegative solution is in fact strictly positive.

Two functions $u, v \in C^{1, \alpha}(\bar{\Omega})$ satisfy the inequality

$$
-\Delta_{p} u-f(u) \leq-\Delta_{p} v-f(v) \text { weakly on } \Omega
$$

Received by the editors April 14, 2006 and, in revised form, June 19, 2006.

2000 Mathematics Subject Classification. Primary 35J70; Secondary 35B05.

Key words and phrases. $p$-Laplace operator, geometric and qualitative properties of the solutions, comparison principle.

Supported by MURST, Project "Metodi Variazionali ed Equazioni Differenziali Non Lineari".

(C)2007 American Mathematical Society

Reverts to public domain 28 years from publication 
if and only if

$$
\int_{\Omega}|\nabla u|^{p-2} \nabla u \cdot \nabla \psi d x-\int_{\Omega} f(u) \psi d x \leq \int_{\Omega}|\nabla v|^{p-2} \nabla v \cdot \nabla \psi d x-\int_{\Omega} f(v) \psi d x
$$

for every $\psi \in W_{0}^{1, p}(\Omega)$ such that $\psi \geq 0$ a.e. Throughout this paper we will assume

$$
(A)_{p}\left\{\begin{array}{l}
\text { both } u \text { and } v \text { are nonnegative on } \Omega, \\
\text { either } u \text { or } v \text { solves problem (1.2), } \\
-\Delta_{p} u-f(u) \leq-\Delta_{p} v-f(v) \quad \text { weakly on } \Omega .
\end{array}\right.
$$

We say that a Strong Comparison Principle (SCP for short) holds for two functions $u, v \in C^{1, \alpha}(\bar{\Omega})$ satisfying $(A)_{p}$ if from the inequalities

$$
u \leq v \quad \text { on } \Omega
$$

we can infer the alternative

$$
u<v \text { on } \Omega \text { unless } u \equiv v \text { on } \Omega .
$$

We want to prove that, under suitable boundary conditions, such an SCP holds.

The novelty of the paper is that $f$ can be a sign changing nonlinearity. For example, our assumptions allow us to consider nonlinearities such as

$$
f(s)=s^{q}-\lambda s^{p-1} \quad(\text { with } q>p-1) .
$$

Even when $f$ has definite sign, it is well known that this is a hard task due to the nonlinear degenerate nature of the p-Laplace operator. In fact, comparison principles are not equivalent in this case to maximum principles as for the case of linear operators. We refer the readers to [10] and the references therein for an interesting overview on this topic, and we recall here some known results.

In [3] it is proved that, if $f$ is locally Lipschitz, a Strong Comparison Principle holds in any connected component of $\Omega \backslash Z_{u, v}$ where $Z_{u, v} \equiv\{x \in \Omega \mid \nabla u(x)=$ $0=\nabla v(x)\}$. In [7] it is proved that, if $f$ is positive and nondecreasing, a Strong Comparison Principle holds assuming that $u, v$ are both solutions of problem (1.2) or assuming as the boundary condition in (1.2) that $u<v$ on $\partial \Omega$. The results in [7] have been recently extended to a more general class of operators in [9, where also some interesting estimates on the set of possible touching points are proved. The assumptions of Theorem 1.3 in [9] are equivalent, in our context, to assuming that $f$ is positive and nondecreasing. Also, we point out some interesting results in [1, 2] where the case of solutions of (1.2) is considered and a Strong Comparison Principle is proved for a particular class of problems involving nonlinearities that do not change sign 1

Some details of our proofs are similar to the ones in [1, 2]. In particular, we point out that we will use a Divergence Theorem stated and proved in [2], together with some regularity results from [4]. The crucial tool anyway is a general result recently obtained in [5] where the case of positive nonlinearities is considered. Here we adapt Theorem 1.4 in 5 , for future use.

\footnotetext{
${ }^{1}$ The nonlinearities considered in 10 2] could change sign if the solutions $u, v$ change sign. Anyway this does not occur since the authors show that the solutions are nonnegative.
} 
Lemma 1.1. Assume $\frac{2 N+2}{N+2}<p \leq 2$ or $p \geq 2$. Let $u, v \in C^{1, \alpha}(\bar{\Omega})$ satisfy $(A)_{p}$ and $f$ satisfy the following hypothesis:

$\left(f_{1}\right) \quad f$ is continuous on $[0,+\infty)$,

$\left(f_{2}\right) \quad f$ is locally Lipschitz continuous on $(0,+\infty)$.

Assume that $u$ is a solution of (1.2) in $\Omega$ and assume that $f(u)$ has a definite sign on a domain $\Omega^{\prime} \subseteq \Omega$ (let us say $f(u)>0$ ); if $u \leq v$ and $u \neq v$ in $\Omega^{\prime}$, then $u<v$ in $\Omega^{\prime}$.

The same result follows assuming that $v$ is a solution of (1.2) in $\Omega$ and $f(v)$ has a definite sign on $\Omega^{\prime}$.

Remark 1.2. The restriction $p>\frac{2 N+2}{N+2}$ allows $|\nabla u|^{p-2}$ to be in $L^{1}(\Omega)$ (in [4] see Theorem 2.3). Lemma 1.1 follows from Theorem 1.4 in [5] by simple considerations. In Theorem 1.4 of [5] only the assumption $f(u)>0$ is considered, however it is clear from its proof that the assumption $f(u)<0$ is equivalent to the assumption $f(u)>0$. The statement of Lemma 1.1 is a local version of Theorem 1.4 in [5] since it holds in any domain $\Omega^{\prime} \subseteq \Omega$. Looking at the proof of Theorem 1.4 in [5] this causes only that a local version of Theorem 2.1 in [5] (see also Theorem 1.1 in [4]) is needed. The latter can be found in [11.

The aim of this paper is to deal with sign changing nonlinearities. More precisely, we keep hypothesis $(A)_{p},\left(f_{1}\right),\left(f_{2}\right)$ without assuming that $f(u)$ or $f(v)$ has definite sign. We simply assume

$$
\left(f_{3}\right) \quad f(t) \begin{cases}=0 & \text { if } t=0 \text { or } t=k>0, \\ <0 & \text { if } t \in(0, k), \\ >0 & \text { if } t \in(k,+\infty),\end{cases}
$$

$\left(f_{4}\right) \quad f$ is nondecreasing on some open interval $I_{k}$ containing $k$.

We prove the following

Theorem 1.3. Assume $\frac{2 N+2}{N+2}<p \leq 2$ or $p \geq 2$. Let $u, v \in C^{1, \alpha}$ satisfy $(A)_{p}$ with $f$ fulfilling $\left(f_{1}\right),\left(f_{2}\right),\left(f_{3}\right),\left(f_{4}\right)$, and assume that $u \leq v$ in $\Omega$. Then, if $u<v$ on $\partial \Omega$, it follows

$$
u<v \text { in } \Omega \text {. }
$$

Theorem 1.4. Assume $\frac{2 N+2}{N+2}<p \leq 2$ or $p \geq 2$. Let $u, v \in C^{1, \alpha}$ both satisfy (1.2) with $f$ fulfilling $\left(f_{1}\right),\left(f_{2}\right),\left(f_{3}\right),\left(f_{4}\right)$, and assume that $u \leq v$ in $\Omega$. Then, if $u \equiv v \equiv 0$ on $\partial \Omega$, the following alternative holds:

$$
u<v \text { in } \Omega \quad \text { or } \quad u \equiv v \text { in } \Omega \text {. }
$$

\section{Proof of Theorem 1.3}

Let us consider the set where $u$ and $v$ possibly coincide:

$$
C_{u, v}=\{x \in \Omega: u(x)=v(x)\} .
$$

We want to show that $C_{u, v}=\emptyset$. By contradiction, we assume that the closed set $C_{u, v}$ is not empty. This, under our hypothesis, equals $\partial C_{u, v} \neq \emptyset$. 
2.1. Step 1. We claim that at each $x \in \partial C_{u, v}$ we have $u(x)=k$. We already know that $u \equiv v>0$ on $C_{u, v} \supset \partial C_{u, v}$ since either $u$ or $v$ is a solution of problem (1.2). Assume by contradiction that there exists some $\bar{x} \in \partial C_{u, v}$ such that $u(\bar{x}) \neq k$. By hypothesis $\left(f_{3}\right)$, we have $f(u(\bar{x})) \neq 0$. Without loss of generality we can consider $f(u(\bar{x}))>0$, and $u$ as a solution of problem (1.2); in this case we can find an open ball $B\left(\bar{x}, r_{\bar{x}}\right)$ centered at $\bar{x}$ such that $f(u)>0$ on $B\left(\bar{x}, r_{\bar{x}}\right)$. Since $\bar{x} \in \partial C_{u, v}, u$ can not coincide with $v$ on the whole $B\left(\bar{x}, r_{\bar{x}}\right)$, thus we can apply Lemma 1.1 getting $u<v$ on $B\left(\bar{x}, r_{\bar{x}}\right)$, and this contradicts the hypothesis $u(\bar{x})=v(\bar{x})$.

2.2. Step 2. By assuming $C_{u, v} \neq \emptyset$, the function $\operatorname{dist}\left(x, C_{u, v}\right)$ is well defined at each $x \in \Omega$ and we can consider the open set

$$
\left.C_{u, v}^{\epsilon}=\left\{x \in \Omega: \operatorname{dist}\left(x, C_{u, v}\right)<\epsilon\right\} \quad \text { (where } \epsilon>0\right) .
$$

Since $u \equiv v \equiv k$ on $\partial C_{u, v}$, we can claim that there exists a $\bar{\epsilon}>0$ such that

$$
\forall x \in C_{u, v}^{\bar{\epsilon}} \backslash C_{u, v} \quad u(x) \in I_{k} \quad \text { and } \quad v(x) \in I_{k} .
$$

On the contrary we would have that

$$
\forall \epsilon>0 \exists x_{\epsilon} \in C_{u, v}^{\epsilon} \backslash C_{u, v} \quad u\left(x_{\epsilon}\right) \notin I_{k} \quad \text { or } \quad v\left(x_{\epsilon}\right) \notin I_{k} .
$$

By choosing $\epsilon=\frac{1}{n}$ there would exist a sequence $\left(x_{n}\right)$ such that

$$
x_{n} \in C_{u, v}^{\frac{1}{n}} \backslash C_{u, v} \quad u\left(x_{n}\right) \notin I_{k} \quad \text { or } \quad v\left(x_{n}\right) \notin I_{k} .
$$

From this sequence we could extract a subsequence $\left(x_{n^{\prime}}\right)$ such that

$$
x_{n^{\prime}} \in C_{u, v}^{\frac{1}{n^{\prime}}} \backslash C_{u, v} \quad w\left(x_{n^{\prime}}\right) \notin I_{k}
$$

where $w$ would be either $u$ or $v$. As $\Omega$ is bounded we could extract from $\left(x_{n^{\prime}}\right)$ a subsequence $\left(x_{n^{\prime \prime}}\right)$ that would necessarily converge to some point $z \in \partial C_{u, v}$ where $w(z)=k$. But this would end the contradiction $w\left(x_{n^{\prime \prime}}\right) \rightarrow k$ and $w\left(x_{n^{\prime \prime}}\right) \notin I_{k}$.

2.3. Step 3 [Contradiction]. By construction we have that $u<v$ on $\partial C_{u, v}^{\bar{\epsilon}}$. As $\partial C_{u, v}^{\bar{\epsilon}}$ is compact, there exists some $\rho>0$ such that $u+\rho<v$ on $\partial C_{u, v}^{\bar{\epsilon}}$. Let us consider the function $w_{\rho}: \bar{\Omega} \rightarrow[0,+\infty)$ defined as follows:

$$
w_{\rho}= \begin{cases}(u+\rho-v)^{+} & \text {on } C_{u, v}^{\bar{\epsilon}}, \\ 0 & \text { on } \bar{\Omega} \backslash C_{u, v}^{\bar{\epsilon}} .\end{cases}
$$

Since $u+\rho<v$ on $\partial C_{u, v}^{\bar{\epsilon}}$, we have that $w_{\rho} \in W_{0}^{1, p}(\Omega)$ and

$$
\nabla w_{\rho}= \begin{cases}\nabla u-\nabla v & \text { where } w_{\rho}>0 \\ 0 & \text { elsewhere }\end{cases}
$$


As $w_{\rho}$ is a test function, we can use it in (1.1) obtaining 2

$$
\begin{aligned}
\int_{\Omega}|\nabla u|^{p-2} \nabla u \cdot \nabla w_{\rho} & =\int_{\Omega} f(u) w_{\rho} \quad\left\langle\text { since } w_{\rho}=0 \text { on } \Omega \backslash C_{u, v}^{\bar{\epsilon}}\right\rangle \\
& =\int_{C_{u, v}^{\bar{\epsilon}}} f(u) w_{\rho} \\
& =\int_{C_{u, v}^{\bar{\epsilon}} \backslash C_{u, v}} f(u) w_{\rho}+\int_{C_{u, v}} f(u) w_{\rho} \\
& =\int_{C_{u, v}^{\bar{\epsilon}} \backslash C_{u, v}} f(u) w_{\rho}+\int_{C_{u, v}} f(v) w_{\rho} \\
& \leq \int_{C_{u, v}^{\bar{\epsilon}} \backslash C_{u, v}} f(v) w_{\rho}+\int_{C_{u, v}} f(v) w_{\rho} \\
& =\int_{C_{u, v}^{\bar{\epsilon}}} f(v) w_{\rho}=\int_{\Omega} f(v) w_{\rho} \\
& \left\langle\operatorname{recall}(A)_{p} \text { and } u \text { is a solution of (1.2) }\right\rangle \\
& \leq \int_{\Omega}|\nabla v|^{p-2} \nabla v \cdot \nabla w_{\rho}
\end{aligned}
$$

that is,

$$
\begin{aligned}
\int_{\Omega}\left(|\nabla u|^{p-2}\right. & \left.\nabla u-|\nabla v|^{p-2} \nabla v\right) \cdot \nabla w_{\rho} \\
& =\int_{\left\{w_{\rho}>0\right\}}\left(|\nabla u|^{p-2} \nabla u-|\nabla v|^{p-2} \nabla v\right) \cdot(\nabla u-\nabla v) \leq 0 .
\end{aligned}
$$

By recalling (see for example [3]) that there exists some positive constant $C_{p}$ such that for each $\eta, \eta^{\prime} \in \mathbb{R}^{N}$

$$
\left(|\eta|^{p-2} \eta-\left|\eta^{\prime}\right|^{p-2} \eta^{\prime}\right) \cdot\left(\eta-\eta^{\prime}\right) \geq C_{p}\left(|\eta|+\left|\eta^{\prime}\right|\right)^{p-2}\left|\eta-\eta^{\prime}\right|^{2}
$$

we get

$$
C_{p} \int_{\left\{w_{\rho}>0\right\}}(|\nabla u|+|\nabla v|)^{p-2}|\nabla u-\nabla v|^{2} \leq 0 .
$$

This implies that $u-v$ equals some constant on $\left\{w_{\rho}>0\right\}$, that is, $w_{\rho}$ is a constant on $\left\{w_{\rho}>0\right\}$. By continuity of $w_{\rho}$ this constant must be zero since $w_{\rho}=0$ on $\partial C_{u, v}^{\bar{\epsilon}}$. Thus, we have that $w_{\rho} \equiv 0$ in $C_{u, v}^{\bar{\epsilon}}$, that is,

$$
\left.u+\rho \leq v \quad \text { on } C_{u, v}^{\bar{\epsilon}} \quad \text { (i.e. } u<v \quad \text { on } C_{u, v}^{\bar{\epsilon}}\right),
$$

and this contradicts the fact that $C_{u, v}^{\bar{\epsilon}} \supset C_{u, v} \neq \emptyset$.

\footnotetext{
${ }^{2}$ We put comments between \langle\rangle brackets.
} 


\section{Proof of Theorem 1.4}

Since $u=0$ on $\partial \Omega$ and $u \in C^{1, \alpha}(\bar{\Omega})$, there exists an open neighborhood $U$ of $\partial \Omega$ such that $0<u<k$ on $V=U \cap \Omega$. Since $f(u)<0$ on $V$, there the SCP holds by Lemma 1.1; therefore $u \equiv v$ on $V$ or $u<v$ on $V$. In the latter case we can find a set $\Gamma^{\epsilon}=\{x \in \Omega: \operatorname{dist}(x, \partial \Omega) \geq \epsilon\}$ for a suitable $\epsilon>0$ such that $u<v$ on $\partial \Gamma^{\epsilon}$; exploiting Theorem [1.3, we get $u<v$ on $\Gamma^{\epsilon}$, and therefore $u<v$ on $\Omega$. Thus, in the sequel we will consider the former case $(u \equiv v$ on $V)$ and prove that $u$ must coincide with $v$ on $\Omega$. As in Theorem 1.3. we define $C_{u, v}=\{x \in \Omega: u(x)=v(x)\}$ and $C_{u, v}^{\epsilon}=\left\{x \in \Omega\right.$ : $\left.\operatorname{dist}\left(x, C_{u, v}\right)<\epsilon\right\}$. Let us assume by contradiction that there exists some $x_{0} \in \Omega$ such that $u\left(x_{0}\right)<v\left(x_{0}\right)$. Arguing as in Section 2.2 of the proof of Theorem 1.3. we can always find an $\epsilon$ such that $0<\epsilon<\operatorname{dist}\left(x_{0}, \partial C_{u, v}\right)$ and

$$
\forall x \in C_{u, v}^{\epsilon} \backslash C_{u, v} \quad u(x) \in I_{k} \quad \text { and } \quad v(x) \in I_{k} .
$$

Let us observe that $\left(\Omega \backslash C_{u, v}\right) \cap C_{u, v}^{\epsilon}$ is a nonempty open set and $\partial C_{u, v}^{\epsilon} \backslash \partial \Omega \neq \emptyset$ by the assumption $0<\epsilon<\operatorname{dist}\left(x_{0}, \partial C_{u, v}\right)$. Moreover at each $x \in \partial C_{u, v}^{\epsilon} \backslash \partial \Omega$ we have $u(x)<v(x)$. By compactness of $\partial C_{u, v}^{\epsilon} \backslash \partial \Omega$ and continuity of $u$ and $v$, there exists $\rho>0$ such that $u+\rho<v$ on $\partial C_{u, v}^{\epsilon} \backslash \partial \Omega$. Let us define

$$
w_{\rho}= \begin{cases}(u+\rho-v)^{+} & \text {on } C_{u, v}^{\epsilon}, \\ 0 & \text { on } \Omega \backslash C_{u, v}^{\epsilon} .\end{cases}
$$

We have that $w_{\rho} \in W^{1, p}(\Omega)$ and

$$
\nabla w_{\rho}= \begin{cases}\nabla u-\nabla v & \text { where } w_{\rho}>0 \\ 0 & \text { elsewhere }\end{cases}
$$

Let us observe that $\nabla w_{\rho}=\nabla u-\nabla v=0$ on $\bar{V}$. This allows us to use $w_{\rho}$ "as a test function" even if $w_{\rho} \notin W_{0}^{1, p}(\Omega)$; in fact, we will see that the boundary terms appearing in the Divergence Theorem for $u$ and $v$ coincide.

As pointed out in [5, a $C^{1}$ solution of (1.2), with $f$ as in our hypothesis, belongs to the class $C^{2}(\Omega \backslash Z)$, where $Z=\{x \in \Omega: \nabla u(x)=0\}$; therefore the generalized derivatives of $|\nabla u|^{p-2} u_{x_{i}}$ coincide with the classical ones on $\Omega \backslash Z$. Moreover in [5] it was proved that $|\nabla u|^{p-2} u_{x_{i}} \in W^{1,2}(\Omega)$. Since $w_{\rho} \in W^{1,2}(\Omega)$ we have that $\operatorname{div}\left(w_{\rho}|\nabla u|^{p-2} \nabla u\right) \in L^{1}$. The vector field $w_{\rho}|\nabla u|^{p-2} \nabla u$ belongs to $\left[C^{0}(\Omega)\right]^{N}$, so we can apply the Divergence Theorem as stated in [2] pag.742, obtaining

$$
\int_{\Omega} \operatorname{div}\left(w_{\rho}|\nabla u|^{p-2} \nabla u\right) d x=\int_{\partial \Omega} w_{\rho}|\nabla u|^{p-2} \frac{\partial u}{\partial \nu} d \sigma
$$


Since $\operatorname{div}\left(w_{\rho}|\nabla u|^{p-2} \nabla u\right)=w_{\rho} \operatorname{div}\left(|\nabla u|^{p-2} \nabla u\right)+|\nabla u|^{p-2} \nabla u \cdot \nabla w_{\rho}$ and also $-\operatorname{div}\left(|\nabla u|^{p-2} \nabla u\right)=f(u)$ almost everywhere, we obtain (exploiting as in Theorem 1.3 )

$$
\begin{aligned}
& \int_{\Omega}|\nabla u|^{p-2} \nabla u \cdot \nabla w_{\rho} d x=\int_{\Omega} f(u) w_{\rho} d x+\int_{\partial \Omega} w_{\rho}|\nabla u|^{p-2} \frac{\partial u}{\partial \nu} d \sigma \\
&=\int_{\Omega} f(u) w_{\rho} d x+\int_{\partial \Omega} w_{\rho}|\nabla v|^{p-2} \frac{\partial v}{\partial \nu} d \sigma \\
&=\int_{C_{u, v}^{\epsilon}} f(u) w_{\rho} d x+\int_{\partial \Omega} w_{\rho}|\nabla v|^{p-2} \frac{\partial v}{\partial \nu} d \sigma \\
&=\int_{C_{u, v}^{\epsilon} \cap C_{u, v}} f(u) w_{\rho} d x+\int_{C_{u, v}^{\epsilon} \backslash C_{u, v}} f(u) w_{\rho} d x+\int_{\partial \Omega} w_{\rho}|\nabla v|^{p-2} \frac{\partial v}{\partial \nu} d \sigma \\
&=\int_{C_{u, v}^{\epsilon} \cap C_{u, v}} f(v) w_{\rho} d x+\int_{C_{u, v}^{\epsilon} \backslash C_{u, v}} f(u) w_{\rho} d x+\int_{\partial \Omega} w_{\rho}|\nabla v|^{p-2} \frac{\partial v}{\partial \nu} d \sigma \\
& \leq \int_{C_{u, v}^{\epsilon} \cap C_{u, v}} f(v) w_{\rho} d x+\int_{C_{u, v}^{\epsilon} \backslash C_{u, v}} f(v) w_{\rho} d x+\int_{\partial \Omega} w_{\rho}|\nabla v|^{p-2} \frac{\partial v}{\partial \nu} d \sigma \\
&=\int_{\Omega} f(v) w_{\rho} d x+\int_{\partial \Omega} w_{\rho}|\nabla v|^{p-2} \frac{\partial v}{\partial \nu} d \sigma \\
&=(*) \\
& \int_{\Omega}|\nabla v|^{p-2} \nabla v \cdot \nabla w_{\rho} d x .
\end{aligned}
$$

Arguing as in Theorem 1.3 we conclude the contradiction $w_{\rho}=0$ (that is, $u+\rho \leq$ $v)$ in $C_{u, v}^{\epsilon} \supset C_{u, v} \neq \emptyset$.

Remark 3.1. Further extensions are possible. For example, one may guess that in Theorem 1.4 the thesis is still valid by assuming that $u, v \in C^{1, \alpha}$ simply satisfy $(A)_{p}$, instead of both being solutions of (1.2). This is actually true if the function that is not a solution of (1.2) (let us say $v$ ) shares the same regularity as the solution $u$. In such a case the Divergence Theorem can still be applied to $v$ giving, with $(A)_{p}$, the inequality $\leq$ instead of the equality at the final step $(*)$. However, we skipped such a statement because here shortness and simplicity is our aim.

\section{REFERENCES}

1. M. Cuesta, P. Takàc, A Strong Comparison Principle for the Dirichlet p-Laplacian Proceedings of the Conference on Reaction diffusion systems (Trieste, 1995), 79-87, Lecture Notes in Pure and Appl. Math., 194, Dekker, New York, 1998. MR1472511 (98i:35058)

2. M. Cuesta, P. Takàc, A Strong Comparison Principle for Positive Solutions of Degenerate Elliptic Equations Differential Integral Equations 13 (2000), no. 4-6, 721-746. MR.1750048 (2001h:35008)

3. L. Damascelli, Comparison theorems for some quasilinear degenerate elliptic operators and applications to symmetry and monotonicity results, Ann. Inst. H. Poincaré. Anal. Non Linéaire, 15 (1998), no. 4, 493-516. MR1632933 (99e:35081)

4. L. Damascelli and B. Sciunzi, Regularity, Monotonicity and Symmetry of positive solutions of m-Laplace equations. J. Differential Equations, 206 (2004), no.2, 483-515. MR2096703 (2005h:35116) 
5. L. Damascelli and B. Sciunzi, Harnack Inequalities, Maximum and Comparison Principles, and Regularity of positive solutions of $m$-Laplace equations. Calc. Var. Partial Differential Equations, 25 (2006), no.2, 139-159. MR2188744(2006i:35084)

6. E. DiBenedetto, $C^{1+\alpha}$ local regularity of weak solutions of degenerate elliptic equations, Nonlinear Anal., 7 (1983), no. 8, 827-850. MR0709038(85d:35037)

7. M. Guedda and L. Veron, Quasilinear elliptic equations involving critical Sobolev exponents, Nonlinear Anal., 13 (1989), no. 8, 879-902. MR1009077 (90h:35100)

8. G.M. Lieberman, Boundary regularity for solutions of degenerate elliptic equations, Nonlinear Anal., 12 (1988), no. 11, 1203-1219. MR0969499 (90a:35098)

9. M. Lucia and S. Prashant, Strong comparison principle for solutions of quasilinear equations, Proc. Amer. Math. Soc., 132 (2004), no. 4, 1005-1011. MR2045415 (2005a:35033)

10. P. Pucci, J. Serrin, The strong maximum principle revised, J. Differential Equations, 196 (2004), no. 1, 1-66. MR2025185 (2004k:35033)

11. B. Sciunzi, Some results on the qualitative properties of positive solutions of quasilinear elliptic equations, NoDEA Nonlinear Differential Equations Appl., to appear.

12. P. Tolksdorf, Regularity for a more general class of quasilinear elliptic equations, J. Differential Equations, 51 (1984), 126-150. MR0727034 (85g:35047)

13. J. L. Vazquez, A strong maximum principle for some quasilinear elliptic equations, Appl. Math. Optim., 12 (1984), 191-202. MR0768629 (86m:35018)

Dipartimento di Matematica, Universà di Roma "Tor Vergata”, Via della Ricerca SciENTIFICA

00133 Roma, ItAly

E-mail address: roselli@mat.uniroma2.it

Dipartimento di Matematica, Università di Roma "Tor Vergata”, Via della Ricerca Scientifica, 00133 Roma, Italy

E-mail address: sciunzi@mat.uniroma2.it 